\title{
ICONIS: the window for URBALIS controlled automatic METRO
}

\author{
P. Noury \\ ALSTOM Transport Information Solutions, France
}

\begin{abstract}
ICONIS implements the concept of an Integrated Control Centre (ICC) and federates former legacy systems such as Automatic Train Supervision, Supervisory Control and Data Acquisition, Passenger Information System, Public Address system and Video-Surveillance. ICONIS is the ultimate solution to craft operator missions according to operational considerations and forgetting about technology.

It is built on the latest development of computer technology and available hardware and software for delivered systems lasting over generations of underlying software.

ICONIS SCADA server monitors all the systems which ensure trains' operations - Interlocking, Automatic Train Control, Automatic Train Protection - and it provides the operator with a live dashboard on system statuses to improve operational availability.

ICONIS incorporates advanced functions for automatic metro to enhance passenger safety. A function monitors platforms to detect passengers on the track and interacts with ATC and video-surveillance systems avoiding high investment in platform screen doors.

Furthermore, ICONIS provides a very efficient operator interface with the Time Distance Graph graphical function and supports operator decisions via Conflict Detection and Solving function.

ICONIS takes care of the renovation project and offers a very modular approach in order to fit exactly to the function to be upgraded. It builds a new base that is suited for further gradual renovation of the other functions aiming at a fully Integrated Control Centre. Whatever the metro system, ICONIS is the solution with the support of URBALIS.
\end{abstract}

Keywords: integrated control centre. 


\section{Introduction}

ALSTOM's ICONISTM implements the concept of Integrated Control Centre (ICC) as part of the URBALIS solution to monitor and control transit rail applications.

URBALISTM is the advanced signalling solutions for all metro and transit rail applications. This solution uses Communication Based Train Control (CBTC) and addresses driver and driverless projects.

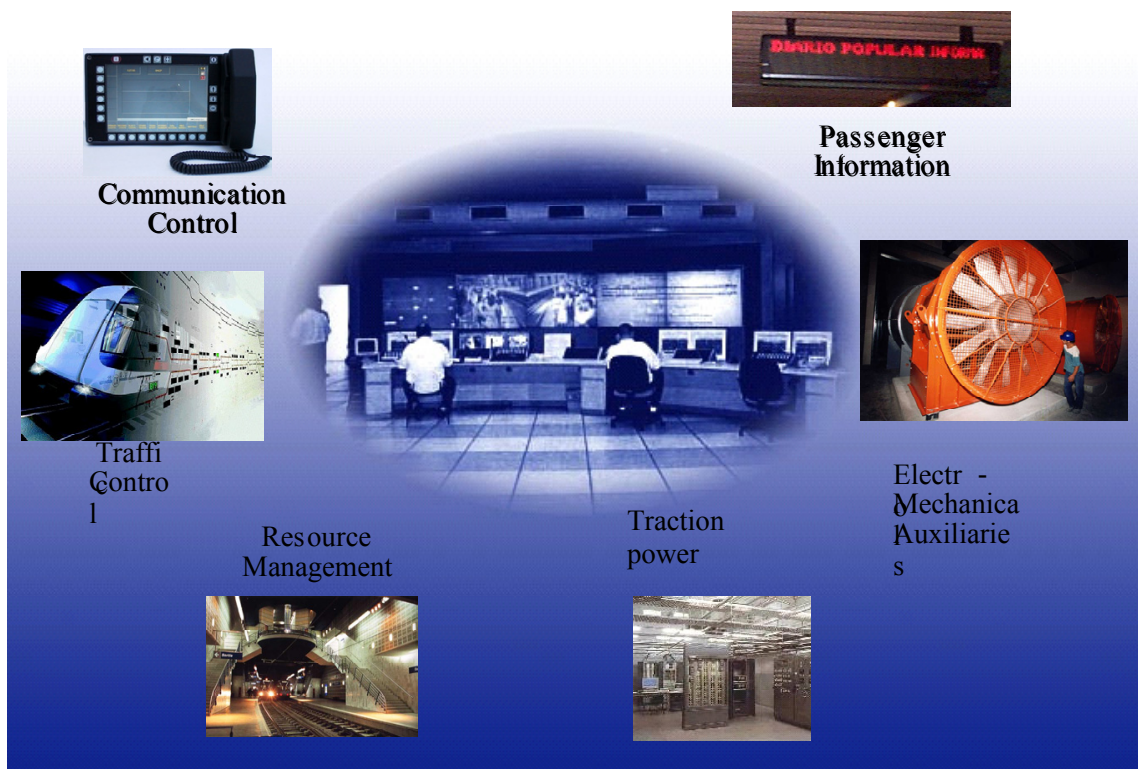

Figure 1: Integrated control centre.

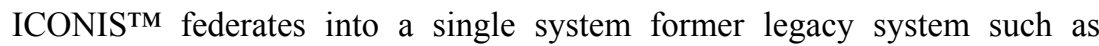
Automatic Train Supervision (ATS) system, Supervisory and Data Acquisition system (SCADA), Passenger Information system (PIS), Public address system (PA), Video Surveillance (CCTV).

This concept provides a smart interface to the control room operator who is able to make the proper decisions in order to run efficiently the metro system and deliver the service required by the customer.

In this sense ICONIS is the single window over the URBALIS solution to monitor and control automatic metros. Through this window, information of different sources and natures may be aggregated together to display the current situation in one single screen.

\section{ICONIS building blocks}

ICONIS provides a single Human to Machine Interface, based on highly flexible graphic mimic diagrams. It also provides a common alarm management system 
in order to build a consistent historian of events for on-line analysis and post mortem investigations.

It is both modular and scalable so that it may only implement one or few functions up to the complete package. It is based on 5 basic products suites:

- $\mathrm{S} 2 \mathrm{~K}$ which encompasses the basic mechanisms for monitoring and control

- Automatic Train Supervision functions

- Field level equipment based on market available software and hardware

- Engineering tools for data base and HMI configuration and simulation tools for testing and training

- Infra ICC for communication management and decision support

These 5 functions may be assembled according to different standard configurations and further called:

- ATS (Supervision of Traffic) when most of the functions are addressing train management,

- SCADA (Supervision of infrastructure) when most of the functions are addressing the management of the infrastructure.

- ICC (Integrated Control Centre) when the above are covered and additional functions are implemented to manage incidents, passengers, resources such as rolling stocks or crews.

When additional functions are needed, they can be added easily to ICONIS standard platform thanks to the use of Window underlying technologies.

This architecture illustrates the concept of software back plane and provides for the unique advantage that new functions do not require re-testing the existing ones. All the functions are fully independent and solely interfaced with the database.

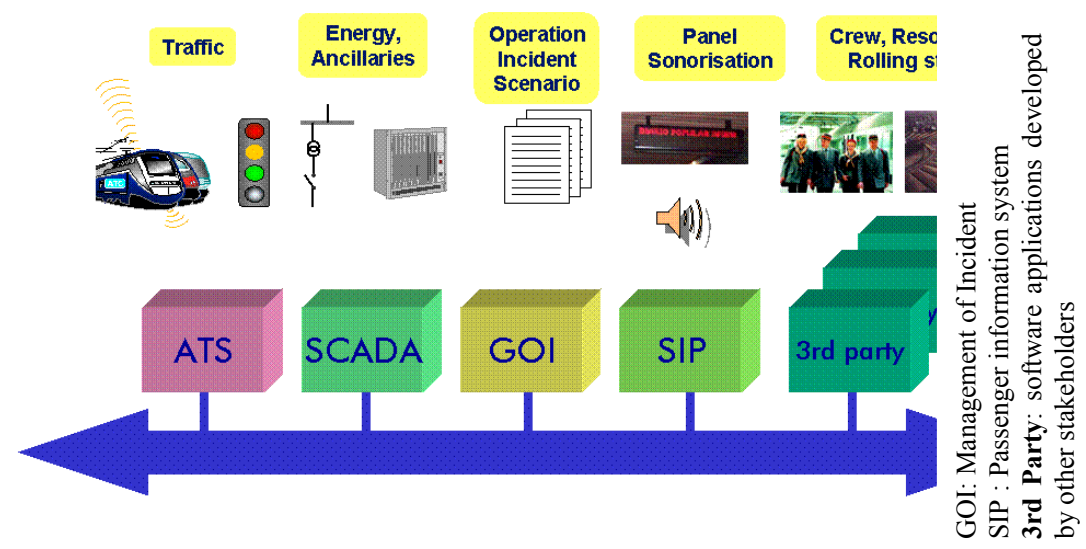

Figure 2: $\quad$ ICONIS ICC software architecture.

\section{Basic technology choices}

The ALSTOM assumptions are based on the availability on the market of a wide range of computers with multiple processors capability together with operating 
systems providing a full library of functions. The drawback of these features is the rapid change of the technology much faster than the evolution of the railway market. Then ICONIS is based on open technologies allowing underneath hardware and middleware to evolve without questioning the application functions and features.

The architecture selected is the classical 3-tiers well known in information technology. The advantages are well known, but specifically for railway application, it provides:

- Applications independence

- Client access to all the application data whatever they are located

- Consistent integration as data are modelled through object classes known across the system

- Server redundancy

- Multiple simultaneous clients

Furthermore the field information is represented along with a common database compliant to OPC (OPen Connectivity). This database supports the representation of each and any railway device. OPC is a well known developed data base very extensively used in industry and supported by multiple software editors (see www.OPCfoundation.org).

This has led to the concept of software components. A component is a software piece implementing an elementary function of the ATS and SCADA domain. The component is interfaced with the database only.

Each component is fully validated and tested and can be considered as an off the self software.

The components may be of very different nature:

- Driver that adapts an external communication protocol to the data base

- The alarm management function

- The signalling rules verification function

- The reporting function

In the other end the concept of software backplane has been implemented. It encapsulates the object oriented infrastructure and the OPC database. It provides all the resources expected by the software components in a common way.

Building an application becomes the art of selecting the right set of component to fulfil client requirements and plugging them into the software backplane. A complete application is then implemented without writing a single line of code.

\section{Communication}

ICONIS servers are permanently connected to the URBALIS secure controllers by means of IP communication links.

To support the communication, the devices use the client servers approach but not in a straightforward implementation in order to ensure:

- Independence between the URBALIS network and the ICONIS network

- Make the URBALIS secure controller non sensitive to the number of ICONIS servers 
This implies the implementation of Proxy devices that will behave as client to URBALIS controllers and servers to ICONIS clients. These proxies will also filter the traffic and avoid that the ICONIS intra traffic that carries large information chunks may perturb the cyclic traffic of the URBALIS controllers.

There will be two proxy devices each one looking at one IP net of the URBALIS communication system. Each proxy will be connected to each redundant ICONIS server in order to provide the best availability.

This organisation provides both:

- Seamless integration as the messages are the same along the link

- Flexibility of the architecture to answer the range of user requirements

- Performance, as each network can be used to its optimum load without having to care about the load and QoS (Quality of Service) of the other one.

\section{Monitoring the environment}

\subsection{System monitoring}

ICONIS, as mentioned before, is able to support a SCADA system with the same underlying technology as the ATS. The SCADA servers exhibit the same interface as the ATS servers so that the operator workstations may access any information managed by either server in a transparent way.

Then the SCADA functions for monitoring and controlling the infrastructure can be applied to the system itself. These capabilities provide the operator with a dashboard summarising the system statuses and telling him if the system is able to achieve the required tasks or if it needs service and where.

\subsection{Passengers monitoring}

In the field of automatic metro, it is of essence to ensure the passenger security. In addition to the legacy domains, it is necessary to improve the passenger security at the transfer level for boarding and un-boarding the trains.

Besides the fact that the SCADA is able to manage platform screen doors there is a need for lighter system to perform similar functions in application such as outdoor stations, refurbishment of existing stations or simply to minimise investment.

Platforms will be equipped with dual detection systems capable of detecting a person falling on the track and reporting on time to the Automatic Train Control system. Those systems should be carefully monitored to ensure that proper mitigation is set in case of an incident and that no false alarm occur and perturb the traffic.

This is achieved by a close cooperation between the detection system, the CCTV system and the SCADA Incident Management Function. The principle is that, when an incident is detected, the alarm is sent to both URBALIS controllers and the ICONIS ICC. If a train is approaching, URBALIS will stop the train, if the train is far from approaching then the information is analysed by the operator 
via auxiliary cameras and Public Address system. This may give time for the person to step back to platform or to ask personnel close by to come and rescue the person.

To help the operator the ICONIS ICC will present him the situation in context (where are the trains, presence of personnel at this location...) together with a checklist for launching the mitigation actions. When incident is mitigated, the ICC system will propose a report of it for filing the case and feeding the database for improving the incident procedures.

This illustrates the fact that even if the functions for passenger monitoring are typically under the SCADA scope, their management is the responsibility of the traffic operator principally dealing with ATS.

In conclusion ICONIS breaks the technology barriers between ATS and SCADA and supports the definition of operator mission only based on functional aspects of the train system operations.

\section{ICONIS train functions}

In order to ensure automatic train operation and traffic regulation for URBALIS controlled metro systems, the ICONIS ATS system addresses the following traditional main functions:

- Field equipment management,

- Train describer,

- Train identification,

- On-line timetable management,

- Automatic route setting,

- Automatic train regulation,

- Depot management,

- Support functions (e.g. Alarms \& Events management),

- Off-line functions.

These functions and the data flow between them are illustrated in the following figure.

The Human Machine Interface offers to each of the above mentioned functions, dedicated interfaces which allow the operator to perform the required controls and information monitoring.

All the above listed functions rely on the integration of the ATS with other specialised subsystems and principally the Interlocking and the Automatic Train Control subsystems. The following is set to illustrate the seamless integration between URBALIS and ICONIS.

The Automatic Train Control management function communicates directly with track-side and embedded URBALIS sub-systems in order to:

○ Report the train location and the train status whatever the trains on the line situation,

o Report train emergencies thanks to fire alarm detections or passengers emergency handles; information is used to query the relevant camera in order to visually confirm the issue and trigger dialogue with passengers,

○ Report trackside emergencies thanks to tunnel fire alarm detection, platform monitoring system, platform plungers,

- Collect maintenance information from the on-board and track side equipment concerning principally signalling, ATC, Rolling Stock 


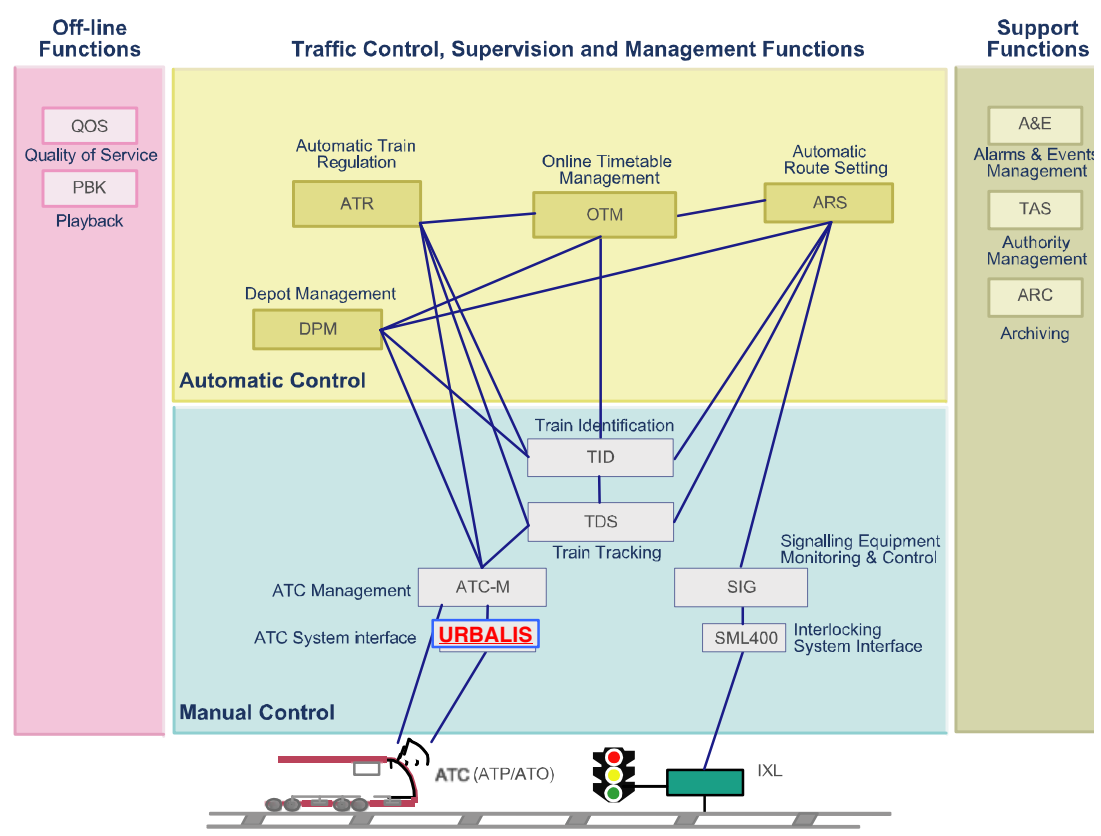

Figure 3.

To achieve its mission, ICONIS implements secure commands when it deals with safety related actions: Temporary Speed Restriction (TSR), Geographical Automatic Mode Authorization (GAMA), Individual Train Automatic Mode Authorization (ITAMA), Track-circuit in/out operation, Initial/Train evacuation reset, Line/siding switching.

Furthermore the URBALIS Management function is in charge of handling information for detailed train operation:

- Train readiness modes,

- $\quad$ Skip-Stop station,

- Departure Time and Arrival Time,

- Hold facilities

(Train, Platform, General Platforms

- Energy saving,

- Change of ends,

- Speed restriction

for un-monitored zone crossing

Connect \& disconnect

The main function of ICONIS ATS is

carriage pairs at depots or sidings.

achieve this, the Train Describer concept has been introduced, which consists in processing trains locations in order to detect relevant events for ATS operation and display trains' movements on HMI.

The train detection movement is based on moving block data provided cyclically by URBALIS ATC system (train identity and location).

As a train progresses along the line, the detection of significant events (e.g. trigger crossing, train arrival, etc) is achieved. These events are sent to the other ATS functions (e.g. Automatic Train Regulation, Automatic Route Setting). 
The operators can follow on HMI the train's progression thanks to the train indicators. Each train indicator is associated to a given portion of track and indicates the identity of the train located within.

\section{Time distance graph and conflict solving}

\subsection{Time distance graphs}

Time Distance Graph (TDG) is a software component, within the range of ICONIS ATS components, intended to help users to monitor and regulate the train traffic circulation.

Connected to the other functionalities of ICONIS ATS (e.g. Timetable Management, Estimated Time of Arrival, Infrastructure Management, etc.), It gives a clear graphical view of the train circulation, infrastructure state, abnormalities and detected conflicts.

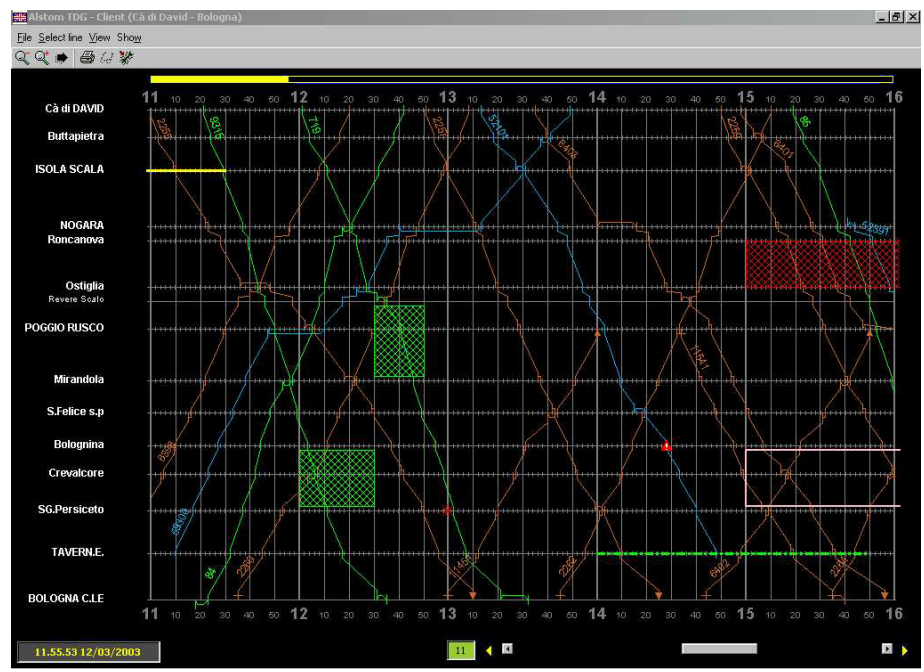

Figure 4.

TDG can be used either in online mode or in off-line mode, in order to plan in advance the train circulation taking account the situation of the infrastructure or temporary modifications to timetables. It is easy to use and highly configurable. It allows the operator to see in advance problems on train circulation in a clear and intuitive way, then to act quickly to solve the problems.

TDG displays:

- Infrastructure provisions (speed restriction or way restriction) by rectangles covering the area involved in the infrastructure provision.

- Abnormalities (general problems that can create any disturbance on the traffic circulation). 
- Conflicts between trains or between a train and the infrastructure by means of an appropriate symbol.

TDG is a convenient summary tool to manage the traffic in a smooth and efficient way

\subsection{Conflict detection and solving}

Each train has its own resources allocated such as infrastructure, rolling stock, crew, etc. These resources are based on daily and current timetable requirements. When disruptions create conflicts in traffic, Conflict Detection and Solving (CDS) checks the resource allocations availability or incompatible usage of resources which lead to conflicts. Then CDS suggests resource allocation (when and what) reassignment and trip modifications to the dispatcher operator to solve these conflicts.

Even if a conflict can be always described by an occupancy resource conflict, it is possible to list four kinds of conflicts:

- Headway conflicts: between two stations, no flanked ways are possible and trains are running in the same direction. Headway time is not respected and trains require the block section line at the same time,

- Cross conflicts: between two stations, one block section line and trains are running in the opposite directions. Trains needs the block section line at the same time,

- Route conflicts: in the station. Trains require two incompatible routes at the same time,

- Platform conflicts: in the station. Trains require the same platform at the same time.

The solution is determined for some (priority mode) or all conflicts (objective mode) detected inside a solving window included in the detection time window.

Each time a conflict is detected, CDS immediately solves it and provides the operator, through either the Time Distance Graph or the Tabular Time Table, with:

- The list of all the conflicts and possible solutions,

- The new proposal trip plan that solves the conflicts.

The solution of a conflict is obtained:

- By modifying the trip of the involved trains in order to avoid the conflict,

- Or selecting alternative resources satisfying the constraints.

\section{Common functions}

ICONIS ICC thanks to its structure is able to collect all the events that occur into the system: Archiving, Playback and Training facilities can be used by operators. 


\subsection{Archiving}

The Archiving function includes two main activities:

- Archiving: storage of data and backup of these data on an external support.

○ Restore: restore of the data from the external support.

\subsection{Playback}

The Playback function allows the data related to a period of operation to be recorded and subsequently replayed. Playback helps in analyzing specific incidents by reviewing the recorded system data (events, alarms, etc).

\subsection{Training facilities}

The training on the ATS functions is feasible on the ATS itself. To achieve this, the ATS is connected to an URBALIS simulator. This simulator will behave as the true system and incorporate train behaviour. Then an instructor station may inject scenario into the simulator for the operator to face real situations and to be trained to make proper decision in time.

\section{Conclusions}

As shown above URBALIS and ICONIS are two sides of the ALSTOM knowledge in transit system control and operations.

There deliberate structure merges all the legacy functions of transit train system together in order to define each operator function based on operational aspects without any technological constraints.

The traditional separation between Train movement, Lateral signalling and infrastructure management has disappeared, but without compromise on security or availability.

New functions have been added to legacy systems such as security of passengers on platform, management of incidents, selective CCTV to facilitate operations

The integration of function including service functions allow to building a single historian database. That database would represent the full history of the transit system and support comprehensive replay of any operation event for understanding incident cause and development, storing the information for authorities investigation, improving the operational rule to minimise the consequences of an incident.

The technology choices provide for a long life of applications even if the underlying computer technology evolves rapidly. In addition the openness of the database (OPC and SQL server) allow for development of specific functions while the system is already up and running. 